\title{
HOLONOMY AND METRIC PROPERTIES OF FOLIATIONS IN HIGHER CODIMENSION
}

\author{
ALEXANDER MORGAN
}

\begin{abstract}
It is well known that a codimension 1 foliation with finite holonomy on a compact manifold must have a bundle-like metric. A counterexample is presented to the higher codimension generalization of this theorem. However, a stronger holonomy restriction (expressed via the Bott connection) is shown to imply the existence of a bundle-like metric.
\end{abstract}

Introduction. The purpose of this paper is to discuss the relationship between holonomy and metric properties for higher codimension foliations. In codimension 1, a theorem of Sacksteder states that if a compact manifold $M$ has a $C^{2}$ foliation $\mathscr{F}$ such that the holonomy of every leaf is finite, then $\mathscr{F}$ has a bundle-like metric. (See $[8$, p. 96].) Note, however, that the proof of this theorem guarantees only a $C^{0}$ bundle-like metric, rather than a smooth one. (See Lawson [5, pp. 409-410].) The straightforward generalization of this theorem is false, and a counterexample is presented in $\S 1$. However, we show in $\$ 2$ that a stronger holonomy restriction, namely that the Bott connection holonomy group be metric preserving, implies there is a bundle-like metric. Preliminary to the proof of this fact, we establish a relation between foliation holonomy and Bott connection holonomy.

I would like to thank H. Shulman and R. Szczarba for very valuable discussions on this material. In particular, Professor Szczarba suggested the value of formalizing the relation between foliation and Bott connection holonomy.

1. Let $M$ be a given manifold. Then there are two equivalent definitions of a foliation $\mathscr{F}$ on $M$, an "integrable distribution" definition (see Bott [1, p. 2]) and a "pseudogroup" definition (see Haefliger [3, p. 368] or [4, p. 146]). There are also two definitions of $\mathscr{F}$ having a bundle-like metric: Reinhart's definition [7, p. 121] which involves the existence of special coordinates for $\mathscr{F}$ as an integrable distribution (see the proof of Theorem 3 in $\$ 2$ of this paper) and Pasternak's definition $[6$, p. 468] which involves a metric restriction on $\mathscr{F}$ as a pseudogroup. It is routine to show the equivalence of these definitions. To minimize the preliminaries, we recall the precise statements of these definitions in the proofs of the theorems as needed, rather than list them here.

The holonomy of a foliation is defined geometrically in [3, p. 380] (pseudogroup definition) and is described by Sacksteder [8, p. 91] from a slightly different (but equivalent) perspective.

We now present a counterexample to the higher codimension generalization

Received by the editors August 22, 1974 and, in revised form, March 10, 1975.

AMS (MOS) subject classifications (1970). Primary 57D30; Secondary 57D15, 57E05.

๑ American Mathematical Society 1976 
of Sacksteder's theorem. For all $q>1$, we will find a compact manifold $M$ with a codimension $q$ foliation $\mathscr{F}$ and all holonomy trivial but having no bundle-like metric.

First we outline the following standard "foliation transverse to fibres" construction. Let $N$ and $F$ be smooth manifolds, and let $\theta: \pi(N) \rightarrow \operatorname{Diff}(F)$ be a smooth action of the fundamental group of $N$ on $F$. If $\tilde{N}$ is the universal cover of $N$, we have a natural action of $\pi(N)$ on $\tilde{N}$ by covering transformations. Therefore, we have an action of $\pi(N)$ on $\tilde{N} \times F$. Note that $\tilde{N} \times F$ has the product foliation with leaves $\tilde{N} \times\{f\}$ for all $f \in F$, and this induces a foliation on $M=\tilde{N} \times F / \pi(N)$, the quotient foliation. The following diagram summarizes:

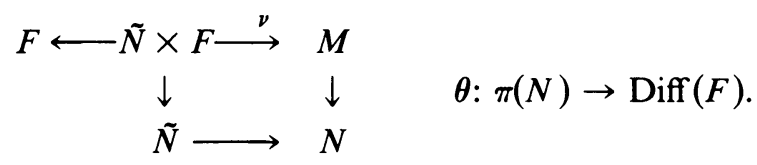

It is not hard to show that given a fibre bundle $F \rightarrow M \rightarrow N$ with a foliation $\mathscr{F}$ on $M$ such that the fibres are transverse submanifolds to the leaves of $\mathscr{F}$, there exists an action $\pi(N) \rightarrow \operatorname{Diff}(F)$ such that the above construction gives the foliation on $M$. (See Thruston [10, pp. 16-17].)

Note that if $L=\nu(\tilde{N} \times\{f\})$ is a leaf, then the holonomy group of $L$ is

$$
H(L)=\{\theta(\sigma) \in \operatorname{Diff}(F) \mid \sigma \in \pi(N) \text { and } \theta(\sigma)(f)=f\} .
$$

Therefore, $H(L)=\{e\}$ for all leaves $L$ if and only if $\Gamma=\theta(\pi(N)) \subseteq \operatorname{Diff}(F)$ acts without fixed points on $F$. (In other words, if $g \in \Gamma$ and $g(x)=x$ for some $x$, then $g=e=$ identity.)

PROPOSITION 1. In terms of the above construction, there is a bundle-like metric for $\mathscr{F}$ on $M$ if and only if there is a Riemannian metric on the manifold $F$ such that $\Gamma$ is a group of isometries.

Proof. Suppose $M$ has a bundle-like metric. Then using Pasternak's definition, we have a $q$-dimensional Riemannian manifold $S$ with metric $\langle$, $\rangle$ such that the distinguished functions for $\mathscr{F}$ take their values in $S ; f_{i}: U_{i} \rightarrow S$ where the $U_{i}$ cover $M$ and $\gamma_{j i}^{x} f_{i}=f_{j}$ in a neighborhood of $x$ in $M$ where $\gamma_{j i}^{x}$ is a local isometry of $S$. The $f_{i}$ and $\langle$, $\rangle$ induce a metric $\langle,\rangle^{\prime}$ on $F$, and since holonomy in $M$ is essentially induced by composing the $\gamma_{j i}^{x}, \Gamma$ must act as isometries on $F$.

Conversely, if there is a metric $\langle,\rangle^{\prime}$ on $F$ such that $\Gamma$ acts as isometries, we may define $\langle$,$\rangle on the normal bundle to the foliation Q \rightarrow M$ to be the metric naturally induced from $\langle,\rangle^{\prime}$ by holonomy translating along paths in leaves from points in $M$ to points in $F$. This $\langle$,$\rangle on Q$ can be extended to $\langle$,$\rangle on$ $T M$, the tangent bundle, obeying the bundle-like metric condition of Reinhart's definition. Q.E.D.

Notice that the above proof is valid without alteration for the more general case in which $M$ is any foliated (codimension $q$ ) manifold with a transverse $q$ dimensional submanifold $F$ intersecting all leaves. ( $\Gamma$ in this general case will be a pseudogroup.)

Now observe that if there is a metric on $F$ such that $\Gamma$ consists of isometries, 
then the action of $\Gamma$ on $F$ must be equicontinuous. (For the definition of equicontinuous, see Furstenberg [2, p. 477] or Sacksteder [8, p. 85].)

Returning to the foliation transverse to fibres construction outlined above, if we can find a fixed point free smooth action $\theta$ of the integers $Z$ on $T^{q}$, the $q$-dimensional torus, which is not equicontinuous, we can then construct a compact $q+1$-dimensional manifold $M$ which is a $T^{q}$ bundle over $S^{1}$ foliated codimension $q$ with trivial holonomy and for which there is no bundle-like metric.

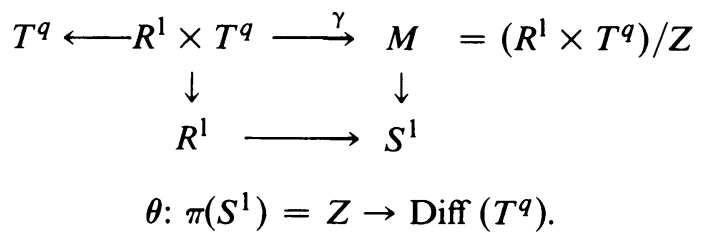

However, in [2, p. 478], Furstenberg has defined such an action of $Z$ on $T^{2}$ which easily extends to a suitable action of $Z$ on $T^{q}$ for $q>1$.

2. We will now establish a relation between Bott connection holonomy and foliation holonomy (Theorem 2) and then prove two metric results (Theorems 3 and 4):

Given a foliation $\mathscr{F}$ on $M$, let $Q \rightarrow M$ denote the normal bundle to $\mathscr{F}$. If $\mathscr{F}$ is given by a $p$-dimensional distribution ( $p$-plane field) $E \subseteq T M$, then $Q$ $=T M / E$. We may take $T M=E \oplus Q$ by identifying $Q=E^{\perp}$. (This last requires a choice of Riemannian metric on $M$.) There is defined on $Q$ a (nonunique) connection $\nabla$, the Bott connection for $\mathscr{F}_{\text {. }}$ (See Bott [1, pp. 32-33].)

Let $M$ be a fixed manifold with codimension $q$ foliation $\mathscr{F}$. Let $Q \rightarrow M$ be the normal bundle to $\mathscr{F}$ and $\nabla$ the Bott connection on $Q \rightarrow M$. Let $x \in M$. Given a loop $\alpha$ in $M$ at $x$, there is an element $\psi(\alpha) \in \operatorname{Hom}\left(Q_{x}\right)$ induced by parallel translation via horizontal lifts of $\alpha$ using $\nabla$. The image of $\psi$, denoted $\bar{H}_{x}$, is called the holonomy group of $\nabla$ at $x$. Identifying $Q_{x}$ with $R^{q}$, we may consider $\bar{H}_{x} \subseteq \mathrm{GL}(q, R)$. Further, we let $\bar{H}_{x}(L)$ denote the subgroup of $\bar{H}_{x}$ consisting of elements induced by loops in $L$ at $x$. Of course, $\bar{H}_{x}$ and $\bar{H}_{x}(L)$ depend on the identification of $Q_{x}$ with $R^{q}$.

Given leaf $L \in \mathscr{F}$ with $x \in L$, the foliation holonomy group $H_{x}(L)$ is a subgroup of Diff $\left(R^{q}, 0\right)$, the germs of diffeomorphisms of $R^{q}$ preserving 0 . It is, in fact, the image of a map $\varphi$ sending homotopy classes of loops in $L$ at $x$ into Diff $\left(R^{q}, 0\right)$. (However, $\varphi$ is only defined up to a choice of an identification of a "transverse $q$-disk at $x$ " with $R^{q}$.) Let $\mu$ : Diff $\left(R^{q}, 0\right) \rightarrow \operatorname{GL}(q, R)$ be defined by $\mu(g)=D g(0)=$ the Jacobian of $g$ at 0 . Call the composition $\mu \circ \varphi$ the "linear holonomy of $\mathscr{F}$ along $L$ (at $x$ )" and the image of $\mu \circ \varphi$, denoted $D H_{x}(L)$, the "linear holonomy group of $\mathscr{F}$ along $L($ at $x)$."

THEOREM 2. The Bott connection holonomy restricted to loops in a leaf $L$ can be identified with the linear holonomy of $\mathscr{F}$ along $L$; i.e. $\psi=\mu \circ \varphi$ and $\bar{H}_{x}(L)=D H_{x}(L)$ for all $L$.

THEOREM 3. F has a bundle-like metric if and only if there is a Riemannian metric on $M$ compatible with some Bott connection on $Q \rightarrow M$. 
THEOREM 4. If the Bott connection holonomy group $H_{x_{0}}$ at some point $x_{0}$ is metric preserving, then $\mathscr{F}$ has a bundle-like metric.

Proof of Theorem 2. This proof is essentially a routine (but minute and tedious) examination of the relevant definitions. We will sketch briefly what is involved. First, we use the definition of the Bott connection given in Bott [1, pp. 32-33]. (See the proof of Theorem 3 in this paper.) Then the following can be shown: Let $\alpha:[0,1] \rightarrow L$ be a path in a leaf $L$, and let $\tilde{\alpha}:[0,1] \rightarrow Q \mid L$ be a horizontal lift. If $\alpha(t)=\sum \alpha_{i}(t) \cdot x_{i}$ in local (distinguished) coordinates $x_{1}, \ldots, x_{n}$, then

$$
\tilde{\alpha}(t)=\sum \alpha_{i}(t) \cdot \tilde{x}_{i}+\sum \tilde{a}_{j} \cdot v_{j}
$$

in local coordinates where $\tilde{x}_{i}$ are appropriately chosen lifting the $x_{i}, v_{j}$ for $j_{i}=p+1, \ldots, n=p+q$ are coordinates in the fibre, and the $\tilde{a}_{j}$ are constant.

Now we use Haefliger's geometric interpretation of holonomy [3, p. 380] which involves covering the image of $\alpha$ by a finite sequence of "distinguished neighborhoods" and performing holonomy transformations successively. It is then not hard to see that the Jacobians of these successive transformations can be identified with the local description for the Bott connection holonomy. It is useful here to note the following: The pseudogroup description of $\mathscr{F}$ involves a covering $\left\{U_{i}\right\}$ of $M$ by distinguished neighborhoods and a choice of submersions $f_{i}: U_{i} \rightarrow R^{q}$ and local diffeomorphisms $\gamma_{j i}^{x}: R^{q} \rightarrow R^{q}$ such that if $x \in U_{i} \cap U_{j}$, then $f_{j} \circ \gamma_{j i}^{x}=f_{i}$ in a neighborhood of $x$. It can be shown that the $g_{j i}: U_{i} \cap U_{j} \rightarrow \mathrm{GL}(q, R)$ defined by $g_{j i}(x)=D \gamma_{j i}^{x}(x)$ give a coordinate transformation description of the normal bundle $Q \rightarrow M$. (See Steenrod [9, pp. 14-15].)

The essential invariance of all of the above under the choices involved can also be shown. Q.E.D.

Lemma. Assume $M$ is foliated with smooth foliation $\mathscr{F}$. Let $\beta:[0,1] \rightarrow M$ be a path in a leaf in some distinguished neighborhood $U$ where $\beta(0)=y$ and $\beta(1)=z$. Let $v: U \rightarrow Q$ be a local section in $Q \rightarrow M$, the normal bundle to $\mathscr{F}$. Then $\psi(\beta)(v(y))=v(z)$ where $\psi(\beta): Q_{y} \rightarrow Q_{z}$ denotes parallel translation via $\beta$ using the Bott connection.

The proof of this lemma follows easily from the definition of the Bott connection.

Proof of Theorem 3. (1) First we recall the Reinhart definition of bundlelike metric. Let the foliation $\mathscr{F}$ on $M$ be given by integrable $p$-plane field $E \rightarrow M$ with normal bundle $Q \rightarrow M$. Let $x \in M$. Since $E$ is integrable, there is a neighborhood $U$ of $x$ such that $T M \mid U$ has coordinates $\partial / \partial x_{1}, \ldots, \partial / \partial x_{p}$, $v_{p+1}, \ldots, v_{n}$ where $\partial / \partial x_{1}, \ldots, \partial / \partial x_{p}$ is a basis for $E \mid U$ and $v_{p+1}, \ldots, v_{n}$ is a basis for $Q \mid U$. We may assume

$$
v_{i}=\frac{\partial}{\partial x_{i}}+\sum_{j=1}^{p} b_{i j} \frac{\partial}{\partial x_{j}}
$$

for some $b_{i j}$. 
Then a metric $\langle$,$\rangle on T M \rightarrow M$ is a bundle-like metric if we can cover $M$ by $U$ as above such that

(i) $\left\langle\partial / \partial x_{i}, v_{j}\right\rangle=0$ for all $i \leqslant p, j \geqslant p+1$ (i.e. $\langle$, $\rangle$ respects a splitting $T M=E+Q)$, and

(ii) $\left\langle v_{i}, v_{j}\right\rangle_{y}=\left\langle v_{i}, v_{j}\right\rangle_{z}$ for all $y, z$ in $U$ such that the last $q$ coordinates of $y$ and $z$ relative to $x_{1}, \ldots, x_{n}$ are equal. (The $x_{1}, \ldots, x_{n}$ are "distinguished coordinates" for $\mathscr{F}$ in $U$; the leaves of $\mathscr{F}$ in $U$ are given as solutions to equations $x_{p+1}=$ constant, $\ldots, x_{n}=$ constant .)

(2) Assume $\langle$,$\rangle is a metric on T M=E \oplus Q$ respecting the splitting and compatible with the Bott connection on $Q$. To show that $\langle$,$\rangle is a bundle-like$ metric, it suffices to prove that if $\left(U ; x_{1}, \ldots, x_{n}\right)$ is a distinguished neighborhood for $\mathscr{F}$ with basis $v_{p+1}, \ldots, v_{n}$ for $Q \mid U$, then $\left\langle v_{i}, v_{j}\right\rangle_{y}=\left\langle v_{i}, v_{j}\right\rangle_{z}$ whenever $y, z$ in $U$ have the same last $q$ coordinates relative to $x_{1}, \ldots, x_{n}$.

Let $\alpha$ be a path from $y$ to $z$ in a leaf in $U$. Then, if $\psi(\alpha): Q_{y} \rightarrow Q_{z}$ is the Bott connection holonomy map, we know that $\psi(\alpha) v_{i}(y)=v_{i}(z)$ and $\psi(\alpha) v_{j}(y)$ $=v_{j}(z)$ by the lemma. Therefore, $\left\langle\psi(\alpha) v_{i}(y), \psi(\alpha) v_{j}(y)\right\rangle_{z}=\left\langle v_{i}(z), v_{j}(z)\right\rangle_{z}$. But, by the compatibility of the Bott connection with $\langle$, $\rangle$, we have $\left\langle v_{i}(y), v_{j}(y)\right\rangle_{y}$ $=\left\langle\psi(\alpha) v_{i}(y), \psi(\alpha) v_{j}(y)\right\rangle_{z}$.

(3) Assume $\langle$, $\rangle$ is a bundle-like metric on $T M \rightarrow M$ where $T M=E \oplus Q$. We want some Bott connection $\nabla$ compatible with $\langle$,$\rangle . This compatibility$ can be described in terms of holonomy translation, as in (2) above, or equivalently by the following relation:

$$
A\langle Y, Z\rangle_{x}=\left\langle\nabla_{A} Y, Z\right\rangle_{x}+\left\langle Y, \nabla_{A} Z\right\rangle_{x}
$$

where $A \in \Gamma(T M)=$ vector fields on $M$ and $Y, Z \in \Gamma(Q)=$ cross-sections in $Q, x \in M$. We now sketch a proof of $(*)$.

Let $D$ be the symmetric connection on $T M \rightarrow M$ compatible with $\langle$,$\rangle .$ Define $\nabla^{\prime}=\pi D$ where $\pi: T M \rightarrow Q$ is projection. Then it is easy to see that $\nabla^{\prime}$ is a connection on $Q$ compatible with $\langle$,$\rangle restricted to Q$. We define a Bott connection $\nabla$ by $\nabla_{X} Z=\pi\left[X_{E}, \tilde{Z}\right]+\nabla_{X_{Q}}^{\prime} Z$ where [, ] denotes Lie bracket, $Z \in \Gamma(Q), \tilde{Z} \in \Gamma(T M)$ with $\pi(\tilde{Z})=Z$, and $X_{E}, X_{Q}$ denote projections of $X$ to $E$ and $Q$ respectively. (See Bott [1, pp. 32-33].) Now by expressing the relation (*) in local (Reinhart) coordinates and using the compatibility of $\nabla^{\prime}$ with $\langle$,$\rangle and the bundle-like metric condition, the proof of (*)$ reduces to showing the following:

Let $M$ be foliated with integrable $p$-plane field $E$ and $Q=E^{\perp}$. If $x \in M$, then there is a neighborhood $U$ of $x$ with local sections $\partial / \partial x_{1}, \ldots, \partial / \partial x_{p}$, $v_{p+1}, \ldots, v_{n}$ for $T M|U=E| U \oplus Q \mid U$ such that if $A$ is a vector field in $E \mid U$, then $\left[A, v_{k}\right]$ is in $E \mid U$ for all $k$. (No metric restrictions needed.) The proof is not difficult. Q.E.D.

Proof of TheOREM 4. Assume the foliation $\mathscr{F}$ on $M$ is given by integrable $p$ plane field $E \rightarrow M$, where $T M=E \oplus Q$. Cover $M$ by distinguished neighborhoods $\left(U_{\alpha} ; x_{1}^{\alpha}, \ldots, x_{n}^{\alpha}\right)$ with $\partial / \partial x_{1}^{\alpha}, \ldots, \partial / \partial x_{p}^{\alpha}$ local sections for $E \mid U_{\alpha}$ and $v_{p+1}^{\alpha}, \ldots, v_{n}^{\alpha}$ local sections for $Q \mid U_{\alpha}$ where

$$
v_{i}^{\alpha}=\frac{\partial}{\partial x_{i}^{\alpha}}+\sum_{j=1}^{p} b_{i j} \frac{\partial}{\partial x_{j}^{\alpha}} .
$$


To show there is a bundle-like metric $\langle$, $\rangle$, we must find a metric $\langle$, $\rangle$ on $T M$ respecting the splitting $T M=E \oplus Q$ such that $\left\langle v_{i}^{\alpha}, v_{j}^{\alpha}\right\rangle_{y}=\left\langle v_{i}^{\alpha}, v_{j}^{\alpha}\right\rangle_{z}$ for all $y, z$ in $U_{\alpha}$ with the same last $q$ coordinates relative to $x_{1}^{\alpha}, \ldots, x_{n}^{\alpha}$, for all $i, j \geqslant p+1$, for all $\alpha$.

Now by hypothesis $H_{x_{0}}$ acts as $\langle,\rangle^{\prime}$ isometries for some metric $\langle,\rangle^{\prime}$ on $Q_{x_{0}}$. Define a metric $\langle$,$\rangle on T M$ as follows: Let $x \in M$ and $v, w \in Q_{x}$. Let $\langle v, w\rangle_{x}=\langle\psi(\beta) v, \psi(\beta) w\rangle_{x_{0}}^{\prime}$ where $\beta$ is any path from $x$ to $x_{0}$. By the condition on the holonomy of the Bott connection at $x_{0},\langle$,$\rangle is well defined. Since$ $\psi(\beta): Q_{x} \rightarrow Q_{x_{0}}$ is an isomorphism, $\langle$,$\rangle is a metric.$

The following argument shows that $\langle$,$\rangle obeys the bundle-like metric$ property stated above: Let $y, x \in U_{\alpha}$. Let $\beta$ be a path from $x$ to $y$ and $\gamma$ a path in a leaf in $U_{\alpha}$ from $y$ to $z$. See Figure 1. (The condition that the last $q$ coordinates of $y$ and $z$ are equal means that they are in the same leaf in $U_{\alpha}$.) We want $\left\langle v_{i}, v_{j}\right\rangle_{y}=\left\langle v_{i}, v_{j}\right\rangle_{z}$. But $\left\langle v_{i}, v_{j}\right\rangle_{y}=\left\langle\psi(\beta) v_{i}, \psi(\beta) v_{j}\right\rangle_{x_{0}}^{\prime}$ by definition of $\langle$,$\rangle , and$

$$
\begin{aligned}
\left\langle v_{i}, v_{j}\right\rangle_{z} & =\left\langle\psi(\gamma \beta) v_{i}, \psi(\gamma \beta) v_{j}\right\rangle_{x_{0}}^{\prime}=\left\langle\psi(\gamma) \psi(\beta) v_{i}, \psi(\gamma) \psi(\beta) v_{j}\right\rangle_{x_{0}}^{\prime} \\
& =\left\langle\psi(\beta) v_{i}, \psi(\beta) v_{j}\right\rangle_{x_{0}}^{\prime}
\end{aligned}
$$

by the lemma.

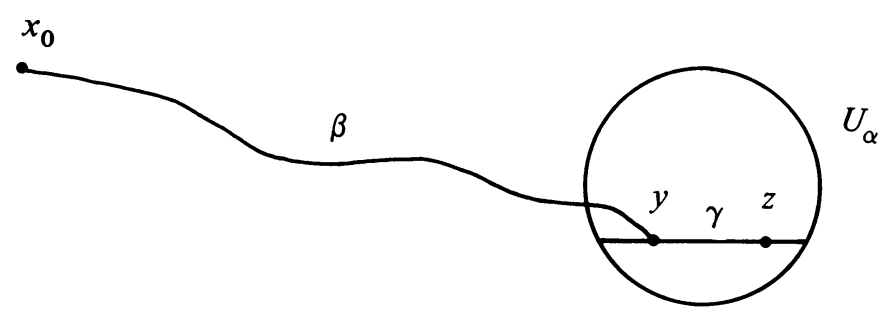

FigURE 1

Q.E.D.

\section{REFERENCES}

1. R. Bott, Lectures on characteristic classes and foliations, Notes by L. Conlon, Mexico, 1971, Lecture Notes in Math., vol. 279, Springer-Verlag, Berlin and New York, 1973.

2. H. Furstenberg, The structure of distal flows, Amer. J. Math. 85 (1963), 477-515. MR 28 \#602.

3. A. Haefliger, Variétés feuilletées, Ann. Scuola Norm. Sup. Pisa (3) 16 (1962), 367-397. MR 32 \#6487.

4. - Homotopy and integrability, Manifolds-Amsterdam 1970 (Proc. Nuffic Summer School), Lecture Notes in Math., vol. 197, Springer-Verlag, Berlin and New York, 1971, pp. 133163. MR 44 \#2251.

5. H. B. Lawson, Foliations, Bull. Amer. Math. Soc. 80 (1974), 369-418.

6. J. S. Pasternack, Foliations of compact Lie group actions, Comment. Math. Helv. 46 (1971), 467-477. MR 45 \# 9353.

7. B. L. Reinhart, Foliated manifolds with bundle-like metrics, Ann. of Math. (2) 69 (1959), 119-132. MR 21 \#6004.

8. R. Sacksteder, Foliations and pseudogroups, Amer. J. Math. 87 (1965), 79-102. MR 30 \#4268. 
9. N. E. Steenrod, The topology of fibre bundles, Princeton Math. Ser., vol. 14, Princeton Univ. Press, Princeton, N.J., 1951. MR 12, 522.

10. W. Thurston, Foliations of 3-manifolds which are circle bundles, Thesis, Univ. of California, Berkeley, Calif., 1972.

Department of Mathematics, Yale University, New Haven, Connecticut 06520

Current address: Department of Mathematics, University of Miami, Coral Gables, Florida 33124 